\title{
Nicht unterlegen, oder doch?
}

\author{
Rainer Lüdtke \\ Karl und Veronica Carstens-Stiftung, Essen, Deutschland
}

Die Debatte währt seit über 200 Jahren, entschieden ist sie noch nicht! Ob die Homöopathie eine wirksame Therapie ist, bleibt auch zwei Jahrhunderte nach ihrer Erfindung (Entdeckung) im Dunkeln. Dabei wird in der Diskussion oft ein wesentliches Detail übersehen bzw. nur am Rande notiert. Wenn wir über die Wirksamkeit der Homöopathie reden, meinen wir eigentlich die Wirksamkeit der homöopathischen Medikamente, oft sogar nur die der Hochpotenzen. Homöopathie ist in der Regel jedoch viel mehr, laut Lehrmeinung gehört eine ausführliche intensive Anamnese unabdingbar zu einer homöopathischen Therapie dazu.

Eine wissenschaftliche Auseinandersetzung mit der Homöopathie müsste demnach auch die Rolle der Anamnese und deren Effekte einbeziehen. In den heute vorliegenden randomisierten Therapiestudien wird genau diese aber technisch ausgeblendet, um sich allein auf die Arzneimittel zu konzentrieren. Auch, oder vielleicht vor allem deshalb, weil dann eine doppelte Verblindung von Arzt und Patient problemlos möglich ist.

Will man dagegen die Anamnese als therapeutisches Konzept mit in die klinische Prüfung integrieren, dann muss man nicht nur auf die Verblindung und damit auch auf eine Placebokontrolle verzichten, sondern in der Regel auch auf die Randomisation: meistens haben die Patienten zu starke Präferenzen, als dass sie einer zufälligen Zuteilung einer Therapie zustimmen könnten [1].

Der Ausweg, den die Homöopathieforschung in den letzten 10 Jahren genommen hat, besteht in nichtrandomisierten Kohortenstudien, in denen homöopathisch behandelte Patienten mit solchen verglichen werden, die gemäss einem konventionellen Konzept therapiert werden. Auch die Forschende KOMPLEMENTÄRMEDIZIN hat wiederholt solche Studien publiziert [2, 3]. Begünstigt wird diese Entwicklung auch durch eine moderne statistische Methodik, die formal korrekte Tests zur Beurteilung der Äquivalenz zweier Therapien bereitstellt, wobei heutzutage in aller Regel nicht auf Äquivalenz, sondern auf Nicht-Unterlegenheit getestet wird.

Doch was sich auf den ersten Blick als «die» Lösung für die Homöopathieforschung anbietet, birgt eine ganze Reihe von Fallgruben. Zunächst einmal werden die Probleme auch in Nicht-Unterlegenheitsstudien nicht kleiner, wenn man auf die Randomisation verzichtet. Zum anderen ermöglicht es gerade die Nicht-Unterlegenheitsstudie, ein positives Ergebnis schon im Studiendesign zu erzwingen. Wie einfach das ist, mögen die folgenden sechs «Vorschläge» illustrieren:

1. Man bestimme den Wert, ab dem die Prüftherapie der Kontrolle nicht unterlegen ist, so grosszügig, dass die Prüftherapie keine Probleme hat, diesen Schwellenwert zu erreichen, selbst wenn sie der Kontrolltherapie unterlegen ist. Ist diese Strategie zu auffällig, z.B. wenn man den Schwellenwert zu weit vom durchschnittlichen Erfolg der Kontrolltherapie platzieren muss, so wechsle man zu nichtparametrischen Verfahren. Eine Unterlegenheitsgrenze auf der Skala eines Wilcoxon-Tests ist fast niemand in der Lage zu interpretieren.

2. Man sorge dafür, dass in beiden Gruppen möglichst wenig Patienten die Therapieempfehlungen befolgen, z.B. durch lange Laufzeiten der Studie oder indem man bei der Aufklärung die potenziellen Nebenwirkungen erwähnt. Dann spiegelt der Therapieerfolg überwiegend den Spontanverlauf der Krankheit wider (vielleicht ergänzt durch unspezifische Wirkungen), und zwar in beiden Gruppen, also Äquivalenz der Therapien.

3. Man sorge für möglichst viele fehlende Werte und ersetze diese durch konservative Strategien, wie sie in Überlegenheitsstudien gebräuchlich und sinnvoll sind (z.B. last-observation-carried-forward). Dieses nivelliert tatsächliche Unterschiede zwischen den Therapien beträchtlich.

4. Man wähle ein Zielkriterium, das weitgehend unabhängig von der Therapie ist, z.B. in akuten Situationen einen Be-

\begin{tabular}{|c|c|}
\hline KARGER & (C) 2006 S. Karger GmbH, Freiburg \\
\hline $\begin{array}{l}\text { Fax +49 } 7614520714 \\
\text { E-mail Information@Karger.de } \\
\text { www.karger.com }\end{array}$ & $\begin{array}{l}\text { Accessible online at: } \\
\text { www.karger.com/fok }\end{array}$ \\
\hline
\end{tabular}

Dipl.-Stat. Rainer Lüdtke 
obachtungszeitraum, in dem die Erkrankung sowieso abgeklungen ist. Ist die Krankheitsaktivität in beiden Gruppen nahe Null, sind beide Therapien äquivalent.

5. Man dosiere die Vergleichstherapie so gering, dass sie ihre vollständige Wirksamkeit nicht entfalten kann.

6. Man gebe sich eine Irrtumswahrscheinlichkeit von $\alpha=5 \%$ vor. Dieses ist auf den ersten Blick unverdächtig, kennt man doch diese Schranke aus vielen Studien der Literatur. Da dort aber üblicherweise zweiseitige Tests verwendet werden, kann man so die Wahrscheinlichkeit verdoppeln, ein (im Sinne der aktiven Therapie) positives Ergebnis zu erzielen.

Dieses gilt natürlich nicht nur für die Homöopathie, sondern auch für konventionelle Therapien. Allerdings sind NichtUnterlegenheitsstudien in der Komplementärmedizin relativ häufiger als bei chemisch definierten Arzneimitteln. In der Akupunktur finden sich z.B. häufig Studien, in denen gleichzeitig eine aktive und eine unbehandelte Kontrollgruppe mitgeführt werden [4]. Wenn wir in Zukunft also weiterhin durchaus sinnvolle und Erfolg versprechende Nicht-Unterlegenheitsstudien durchführen wollen, so müssen wir uns auch gegen die spezifischen Fallstricke wappnen. Nicht unterlegen heisst leider nicht immer wirklich nicht unterlegen, sondern manchmal auch einfach nur suboptimal geplant.

\section{Literatur}

1 Lüdtke R: Therapieforschung in Zeiten der Patientenpräferenz. Z Arztl Fortbild Qualitatssich 2005;99:279-284.

2 Ammerschläger H, Klein P, Weiser M, Oberbaum M: Behandlung von Entzündungen im Bereich der oberen Atemwege - Vergleich eines homöopathischen Komplexpräparates mit Xylometazolin. Forsch Komplementarmed Klass Naturheilkd 2005;12(1):24-31.

3 Weber U, Lüdtke R, Friese KH, Fischer I, Moeller H: A non-randomised pilot study to compare complementary and conventional treatments of acute sinusitis. Forsch Komplementärmed 2002;9(2):99-104.

4 Fink M, Rosted P, Bernateck M, Stiesch-Scholz M, Karst M: Acupuncture in the treatment of painful dysfunction of the temporomandibular joint - a review of the literature. Forsch Komplementarmed 2006;13(2):109-115. 